\title{
Sex differences in discharge therapy in young patients with acute coronary syndrome
}

\section{OFilip Puškarić1 ${ }^{*}$, CNikolina Maglić', OZvonimir Ostojić',, , Olvo Planinc ${ }^{1,2}$, (1) Joško Bulum ${ }^{1,2}$, (CDavor Miličić1,2, (C)Maja Čikeš ${ }^{1,2}$}

'University of Zagreb School of Medicine, Zagreb, Croatia 2University Hospital Centre Zagreb, Zagreb, Croatia

\section{RECEIVED:}

December 14, 2020

ACCEPTED:

December 18, 2020

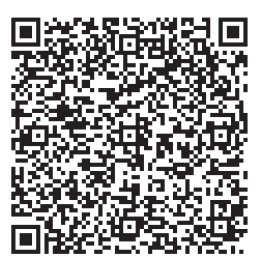

$\square$ Cardiologia Croatica 2021;16(1-2):80.

KEYWORDS: acute coronary syndrome, myocardial infarction, young, therapy.

CITATION: Cardiol Croat. 2021;16(1-2):80-2. | https://doi.org/10.15836/ccar2021.80

*ADDRESS FOR CORRESPONDENCE: Filip Puškarić, University of Zagreb School of Medicine, Šalata 3, HR-10000 Zagreb, Croatia. / Phone: +385-99-7505-240 / E-mail: fpuskaric@gmail.com

ORCID: Filip Puškarić, https://orcid.org/0000-0001-5519-439X • Nikolina Maglić, https://orcid.org/0000-0002-6893-3734 Zvonimir Ostojić, https://orcid.org/0000-0003-1762-9270 • Ivo Planinc, https://orcid.org/0000-0003-0561-6704 Joško Bulum, https://orcid.org/0000-0002-1482-6503 • Davor Miličić, https://orcid.org/0000-0001-9101-1570 Maja Čikeš, https://orcid.org/0000-0002-4772-5549

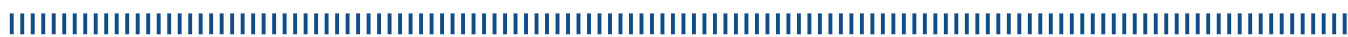

Introduction: Acute coronary syndrome (ACS), including ST-segment elevation myocardial infarction (STEMI), non-ST-elevation myocardial infarction (NSTEMI) and unstable angina pectoris (UA), has been less researched in the subpopulation of young patients. Sex differences in discharge therapy after ACS have been described in older patients as favouring the male sex ${ }^{1}$, and we decided to test this hypothesis in young patients. The term "young" (with regard to ACS) is not uniquely defined, but according to multiple sources, the proposed cut-off age is 45 years ${ }^{2}$.

Patients and Methods: A total of 361 young patients (with an age cut-off of 45 years for men and 55 years for women) with ACS and percutaneous coronary intervention (PCI) performed at the University Hospital Centre Zagreb (UHCZG) between Jan $1^{\text {st }} 2012$ and Jan $1^{\text {st }} 2020$ were enrolled. The patients were divided into the UHCZG group (241 patients discharged home after ACS) and the PCI network group (120 patients transferred to another medical facility after ACS). We explored statistically relevant associations between sex and discharge therapy using the Pearson chi-square test in the UHCZG group, due to the completeness of this dataset.

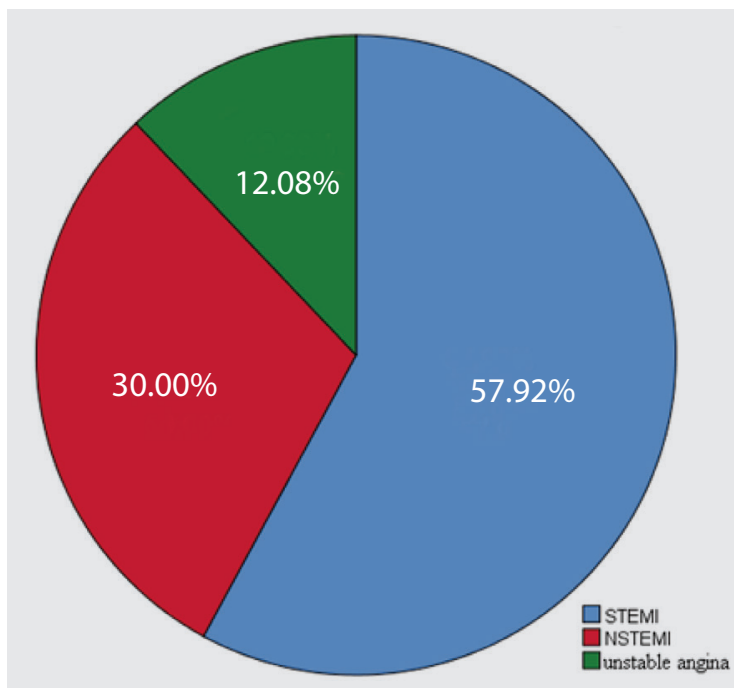

FIGURE 1. Pie chart showing the distribution of types of acute coronary syndrome in the University Hospital Centre Zagreb group.

STEMI - ST-segment elevation myocardial infarction; NSTEMI - non-ST-elevation myocardial infarction.
Results: The mean patient age in the UHCZG group was $44 \pm 7$ years, and 148 (61.4\%) were men. 139 (57.9\%) patients had STEMI, 72 (30.0\%) NSTEMI, and 29 (12.1\%) had UA (Table 1, Figure 1). Female patients had a higher prevalence of hypothyroidism lower diastolic blood pressure at admission and higher LDL values (Table 1). A significantly higher proportion of male patients were prescribed with angiotensin-converting enzyme inhibitors (ACEi) (82.4\% versus $65.6 \%, p=0.003$ ). There were no sex differences in the prescription of other cardiovascular drugs (Table 2).

Conclusion: In a group of young patients with ACS, we found a statistically significant difference between male and female patients regarding the prescription of ACEi, despite a lack of significant difference in systolic blood pressure. Results similar to ours, regarding sex differences in optimal medical therapy, have been described in the literature, including young ACS patients ${ }^{3}$.These results raise concerns regarding potentially negative consequences on the health of young women treated for ACS. 
TABLE 1. Baseline patient characteristics by sex in the University Hospital Centre Zagreb group.

\begin{tabular}{|c|c|c|c|}
\hline Characteristic & UHCZG group - women $(n=93)$ & UHCZG group - men $(n=148)$ & p-value \\
\hline Age, years & $50 \pm 5$ & $41 \pm 4$ & $<0.001$ \\
\hline $\mathrm{BMI}, \mathrm{kg} / \mathrm{m}^{2}$ & $27.9 \pm 6.6$ & $29.9 \pm 5.4$ & 0.018 \\
\hline \multicolumn{4}{|l|}{ ACS type } \\
\hline STEMI & $50(53.8)$ & $89(60.5)$ & $0.298^{*}$ \\
\hline NSTEMI & $29(31.2)$ & $43(29.3)$ & $0.749 *$ \\
\hline Unstable angina & $14(15.1)$ & $15(10.2)$ & $0.263^{*}$ \\
\hline Arterial hypertension & $53(57.0)$ & $78(52.7)$ & 0.516 \\
\hline Diabetes mellitus & $16(17.2)$ & $14(9.5)$ & 0.077 \\
\hline Hypothyroidism & $7(7.6)$ & $2(1.4)$ & 0.013 \\
\hline Dyslipidemia & $47(50.5)$ & $77(52.0)$ & 0.818 \\
\hline \multicolumn{4}{|l|}{ Family history of CVD } \\
\hline CVD at younger age & $10(18.2)$ & $15(15.6)$ & $0.682^{\star \star}$ \\
\hline CVD at older age & $8(14.5)$ & $10(10.4)$ & $0.453^{* *}$ \\
\hline CVD at unknown age & $11(20.0)$ & $11(11.5)$ & $0.153^{\star \star}$ \\
\hline Previous myocardial infarction & $12(12.9)$ & $16(10.9)$ & 0.638 \\
\hline Previous TIA or CVA & $1(1.1)$ & $2(1.4)$ & 0.849 \\
\hline Previous angina pectoris & $32(34.8)$ & $38(25.7)$ & 0.131 \\
\hline \multicolumn{4}{|l|}{ Smoking status } \\
\hline Non-smoker & $20(22.2)$ & $20(14.3)$ & $0.121^{\star}$ \\
\hline Former smoker & $6(6.7)$ & $11(7.9)$ & $0.734^{*}$ \\
\hline Current smoker & $64(71.1)$ & $109(77.9)$ & $0.246^{*}$ \\
\hline \multicolumn{4}{|c|}{ Number of significant coronary stenoses } \\
\hline 0 & $0(0.0)$ & $5(3.4)$ & $0.073^{* \star}$ \\
\hline 1 & $58(63.0)$ & $95(65.1)$ & $0.749 * *$ \\
\hline 2 & $17(18.5)$ & $36(24.7)$ & $0.263^{\star \star}$ \\
\hline 3 & $17(18.5)$ & $10(6.8)$ & $0.006^{* *}$ \\
\hline \multicolumn{4}{|l|}{ Culprit vessel } \\
\hline LAD & $36(39.1)$ & $64(44.1)$ & $0.447^{*}$ \\
\hline LCX & $17(18.5)$ & $28(19.3)$ & $0.873^{\star}$ \\
\hline $\mathrm{RCA}$ & $39(42.4)$ & $48(33.1)$ & $0.147^{*}$ \\
\hline \multicolumn{4}{|l|}{ Dominant coronary artery } \\
\hline Right & $65(83.3)$ & $102(78.5)$ & $0.390^{*}$ \\
\hline Left & $7(9.0)$ & $20(15.4)$ & $0.184^{\star}$ \\
\hline Co-dominance & $6(7.7)$ & $8(6.2)$ & $0.667^{*}$ \\
\hline Systolic BP at admission, $\mathrm{mmHg}$ & $138 \pm 29$ & $142 \pm 25$ & 0.265 \\
\hline Diastolic BP at admission, $\mathrm{mmHg}$ & $85 \pm 16$ & $90 \pm 17$ & 0.011 \\
\hline Heart rate at admission, beats/min & $77 \pm 18$ & $80 \pm 17$ & 0.369 \\
\hline Total cholesterol, mmol/L & $5.15 \pm 1.34$ & $5.07 \pm 1.35$ & 0.706 \\
\hline Triglycerides, mmol/L & $1.77 \pm 0.88$ & $2.04 \pm 1.33$ & 0.094 \\
\hline $\mathrm{HDL}, \mathrm{mmol} / \mathrm{L}$ & $1.17 \pm 0.39$ & $0.96 \pm 0.27$ & $<0.001$ \\
\hline LDL, mmol/L & $3.19 \pm 1.26$ & $3.23 \pm 1.19$ & 0.809 \\
\hline
\end{tabular}

If not otherwise marked, significance determined at $p$-value of 0.05 . ${ }^{*}$ significance of $p$-value $=0.008$ (due to Bonferroni correction) ${ }^{* *}$ significance of $p$-value $=0.006$ (due to Bonferroni correction).

UHCZG - University Hospital Centre Zagreb; BMI - body mass index; ACS - acute coronary syndrome; STEMI - ST-segment elevation myocardial infarction; NSTEM - non-ST-elevation myocardial infarction; CVD - cardiovascular disease; TIA - transient ischemic attack; CVA - cerebral vascular accident; LAD - left anterior descending artery; LCX - left circumflex artery; RCA - right coronary artery; BP - blood pressure; HDL - high-density lipoprotein; LDL - low-density lipoprotein. 
TABLE 2. Sex differences in discharge therapy in the University Hospital Centre Zagreb group.

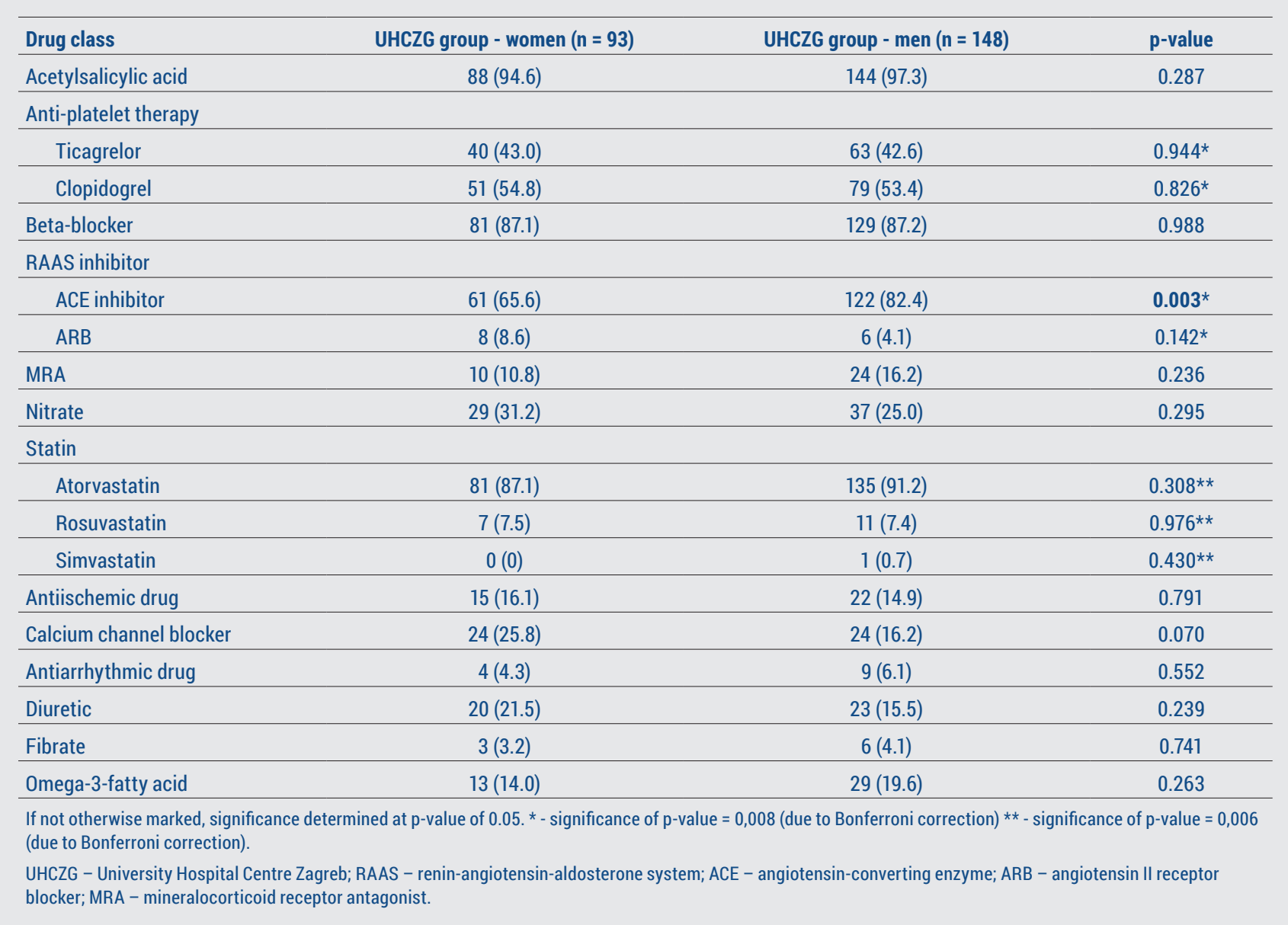

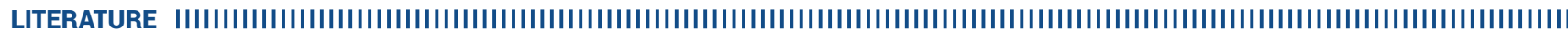

1. Hao Y, Liu J, Liu J, Yang N, Smith SC Jr, Huo Y, et al. Sex Differences in In-Hospital Management and Outcomes of Patients With Acute Coronary Syndrome. Circulation. 2019 Apr 9;139(15):1776-1785. https://doi.org/10.1161/CIRCULATIONAHA.118.037655

2. Hoit BD, Gilpin EA, Henning H, Maisel AA, Dittrich H, Carlisle J, et al. Myocardial infarction in young patients: an analysis by age subsets. Circulation. 1986 0ct;74(4):712-21. https://doi.org/10.1161/01.CIR.74.4.712

3. Davis M, Diamond J, Montgomery D, Krishnan S, Eagle K, Jackson E. Acute coronary syndrome in young women under 55 years of age: clinical characteristics, treatment, and outcomes. Clin Res Cardiol. 2015 Aug;104(8):648-55. https://doi.org/10.1007/s00392-015-0827-2 